\title{
DESIGN AND ANALYSIS OF EDF SCHEDULING Algorithm For A ChOSEn Network TrafFiC Problem: IMPLEMENTATION AND Simulation ANALYSIS
}

\author{
Jude Ighodaro \\ Department Of Computer Science \\ Wroclaw University of Technology, Poland \\ ighodaro@consultant.com,jude.ighodaro@pwr.edu.pl
}

\begin{abstract}
Workstations, CPU etc are in a period of transition, moving from a relatively slow network links and data oriented services to a high set fibre optic services and more diverse services. As such many of the hard and soft real time will not only demand high bandwidth but also a predictable and guarantee Quality of Service (QoS) along side, which is not offered in a best effort network (BE). The provision and terms of $(\mathrm{QoS})$ to real-time applications is a key issue in promising broadband packet switched networks and network of other sorts. Applications such as voice and video typically require QoS guarantees in terms of end-to-end data transfer delays. Supporting the diverse and of course, heterogeneous delay requirements of applications with widely varying characteristics requires packet scheduling schemes more sophisticated than First-In-First-Out (FIFO) at each switch in the network(2) are one of most accepted examples of such scheduling schemes. This points to the convincing need for a QoS, which apportion a high network utilization to be realized.In this paper, we focus on the EDF scheduling scheme, since it is known to provide the optimal delay performance (2), in the deterministic environment, and can for this reason be expected to perform well in the real time setting as well. The large amount of bandwidth as promised by the future network also offer the integration of real time applications, hard or soft.
\end{abstract}

Key words: EDF (Earliest Deadline First), FIFO (First In Fist Out), QoS (Quality Of Service)

\section{Introduction}

\subsection{Overview}

Invariably, non of the early computerized network applications were concerned or sensitive to any of the QoS assurances such as the delay that might arise or the data losses as a result of any unprecedented event. Yes, we had 
internet a number of years ago ,but this type of network was not intended to provide services for those types of data traffic mentioned above, but rather a services that is a called a best effort services. Today the internet offers different types of guarantees or warranties to the different classes of applications using it, these guarantees are called the (QoS) D. Anick, D. Mitra, and M. M. Sondhi.(1982)[1]. The QoS guarantees can be in diverse forms, such as bandwidth, miss ratio in terms of packet loss, total packet delays e.t.c G. de Veciana, C. Courcoubetis, and J. Walrand. CA, (1994) [3]. The applications that convey this type of network, which offers QoS guarantees on the data traffic are of diverse types, some of them are real-time applications in which a rigid QoS guarantees are required from the network, while other applications does not require any guarantees (best-effort traffic),in this regard the network should be capable of providing such guarantees to the various types of traffic that made use of this network. Different technique can perhaps be use to provide this QoS guarantees to the traffic link users.

\section{Simulation Results and Performance Analysis}

\section{$2.1 \mathrm{EDF} / \mathrm{FCFS}$}

To verify the performance of EDF and FCFS, we have simulated EDF and FCFS features, In each simulation, logical channels are randomly generated, in our work we assume poison model packet arrival and other assumptions were also made (chapter 2 and 3). The traffic intensity is increased by increasing the number of logical channels. Such simulations are run 100 times to get the average utilization at different traffic loads. Simulations were performed to analyze delay, throughput, acceptance ratio and deadline miss ratio of the HRT and SRT traffic(EDF, FCFS) by varying the intensity of the two realtime traffic classes respectively. Sporadic traffic channels in the simulator are treated as follows. In order to represent and simulate incoming traffic over the whole period, a arbitrary offset is computed that decides in which time slot in each period a certain channel will be requested for transmission. Our channel comprises of increasing packet load and as such shows the behaviour of the system as represented below

\subsection{Acceptance ratio}

Figure 1 shows how the values of acceptance ratio decreases as the number accepted packets in the requested/available channels increases, it can be seen that at a value of about 40 packets per channels, the acceptance ratio remain about $100 \%$. Which shows that all packets where eventually accepted for transmission. But these ratio decreases as the number of packet requesting 
transmission increases. Our Queues consist of Soft and hard real time queues, FCFS and EDF. Since the utilization, deadline miss ratio and delay are affected by the accepted ratio, G. de Veciana and G. Kesidis (1996). As a result, a reduction in acceptance ratio is to ensure a more feasible schedule and prioritize hard real time task so that they all meet deadlines.

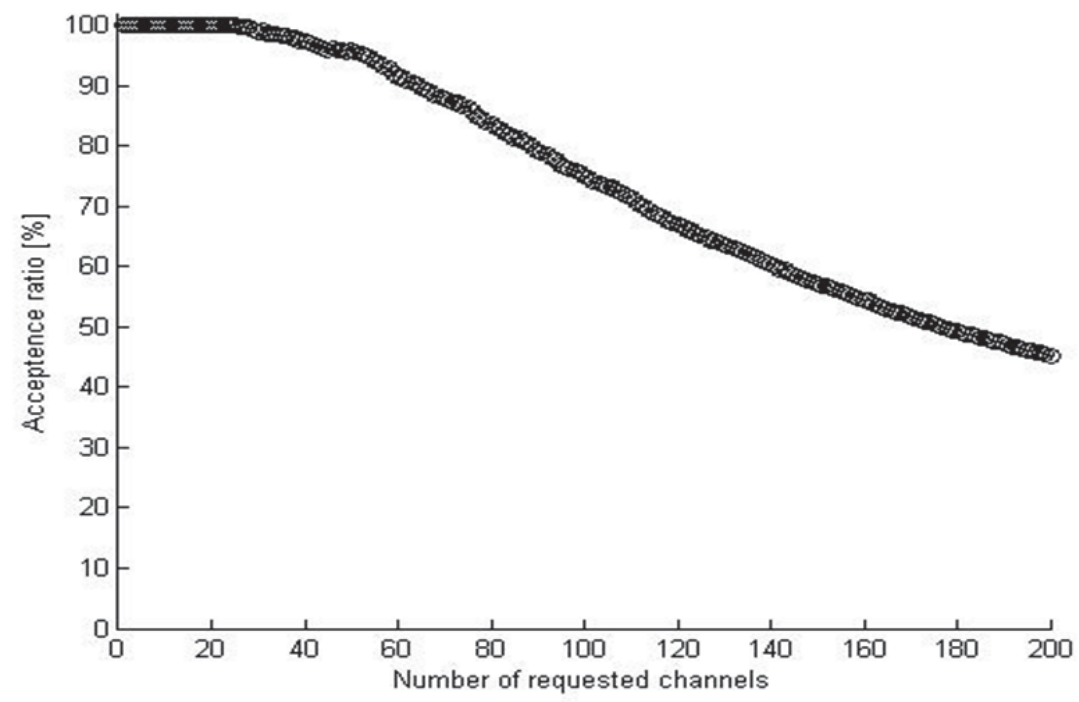

Figure 1. Acceptance ratio

\subsection{Utilization (throughput) of the link analysis}

By utilization (throughput) we mean an average rate of successful message delivery over a communication channel. This data may be delivered over a physical or logical link, or pass through a certain network node. The throughput is usually measured in bits per second (bit/s or bps), and sometimes in data packets per second or data packets per time slot. Figure 1 shows the throughput of the traffic classes, plotted against the logical channel. As the intensity of the traffic increases, its throughput reaches a maximum of about 0.98 and 15 packets per time slot in the channel at its highest traffic intensity (15 packets per period). Due to its high priority, the throughput of the HRT traffic is not affected and remains feasible and schedulable so that the utilization those not exceed 1. Comparing the throughput of both algorithm, according to our analysis, this figure appears to be the same in that the utilization of the link for both classes must not exceed $100 \%$. 


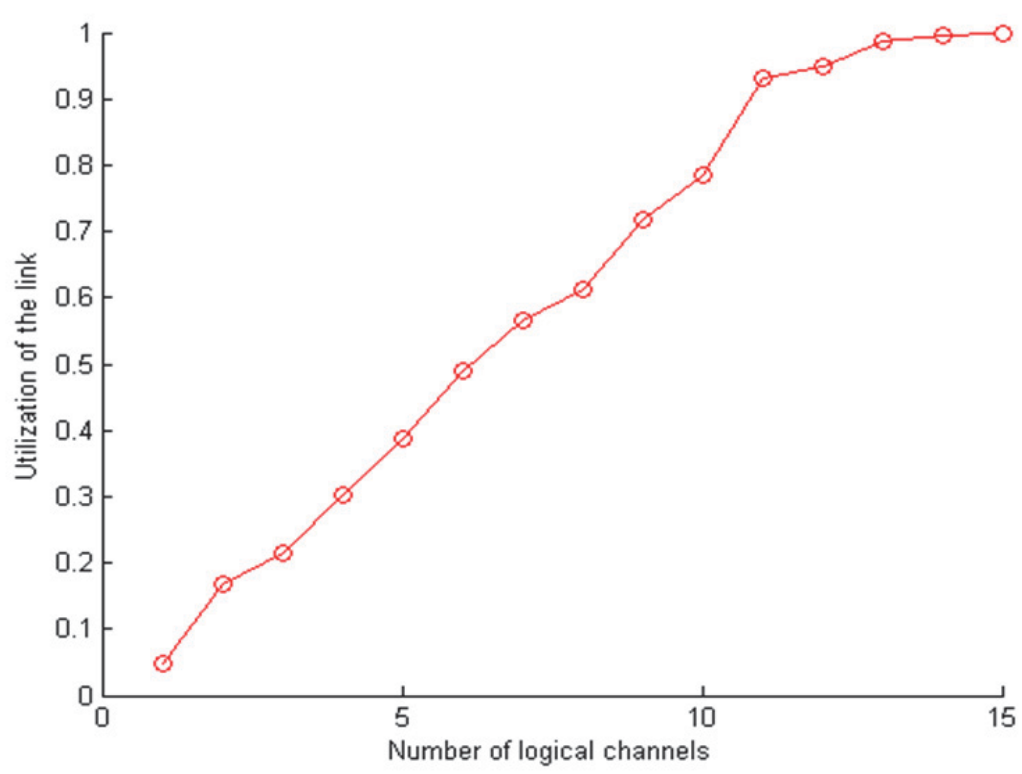

Figure 2. Utilization of the link for DPQ ( Dynamic Priority Queue ) FCFS

Figures 1 and 2 shows how the values of the deadlines influence the utilization.

In the test result, when the test task workload is less than or equal to $100 \%$, both FCFS and the EDF scheduling algorithms can complete tasks; but the CPU efficacious utilization of the EDF algorithm is higher than FCFS , which can save resources, especially when the task workload closes to $100 \%$. Figure 1 also reveals that our system can reach rather high utilization (close to the theoretical utilization upper bound), i.e., utilization reaches $93 \%$ when deadlines are twice the periods (the theoretical utilization bound is $100 \%$ in this case). 


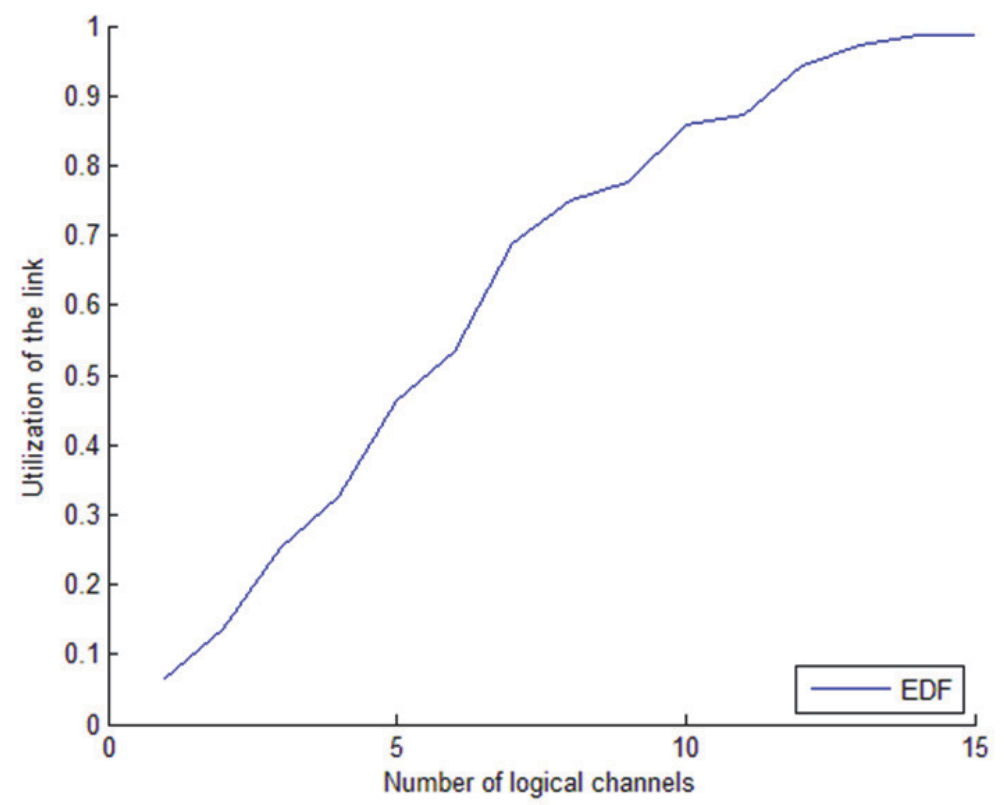

Figure 4. Utilization of the link for EDF DPQ (Dynamic Priority Queue)

The major differences are as follows. Both Figures 3 and 4 visualises the throughput for messages with different deadlines in a network with 4 nodes for a traffic intensity up to 15 packets per node and time slot, i.e. it shows the number of generated packets, which are actually accepted for transmission in each time slot. Independent of the deadline, the throughput of the traffic rises linearly up to a traffic density of 11 and 14 packets per node and time slot for Figures 3 and 4 respectively. The saturation of the network at that point becomes obvious. We can see here that for the static nature of FCFS, the saturation occurred earlier as compared to Dynamic EDF. In that the curves flattens out at a throughput of slightly more than 11 packets per time slot for FCFS. But EDF was able to handle individual packet up to about 14 packets per node before it flatten out and reaches saturation

As expected, throughput varies according to network size. The throughput is proportional to the number of in the system, i.e. proportional to the maximum number of generated packets. Increasing the network size from 8 to 16 nodes for example, results in a doubled throughput; an increase from 8 to 32 , leads to a throughput of four times more packets per time slot. 


\subsection{Average Delay of The link Analysis}

By average delay we mean the amount of individual packet delay over the total delay of the overall systems. Figure 4 shows the average delay for EDF queues plotted against the number of channels. In our model as represented below, each channels are made up of 5, 10, 15 packets per slots of the traffic classes. As the traffic load in the system increases, the traffic experiences a slight increase in delay, while the HRT (EDF) traffic remains at a constant average delay of 10 slots per packet throughout the duration of the simulation. Because of EDF queues, its low priority traffic has to wait for the real-time or HRT traffic classes or higher priority to be sent, which increases its delay considerably.

The behaviour of the delay in Figure 4 can be explained accordingly. Basically this is to understanding of mainly EDF queues or HRT queues. Since we just have an EDF queue, With an increased HRT traffic intensity, the traffic class with the highest priority, HRT traffic, is affected the least, while the remaining other traffic types experience a more significant increase in delay. The average delay for the NRT traffic/FCFS queues is not plot in the figure, as the network already is saturated with NRT traffic which would lead to irregularities in the curve due to insufficient statistical data.

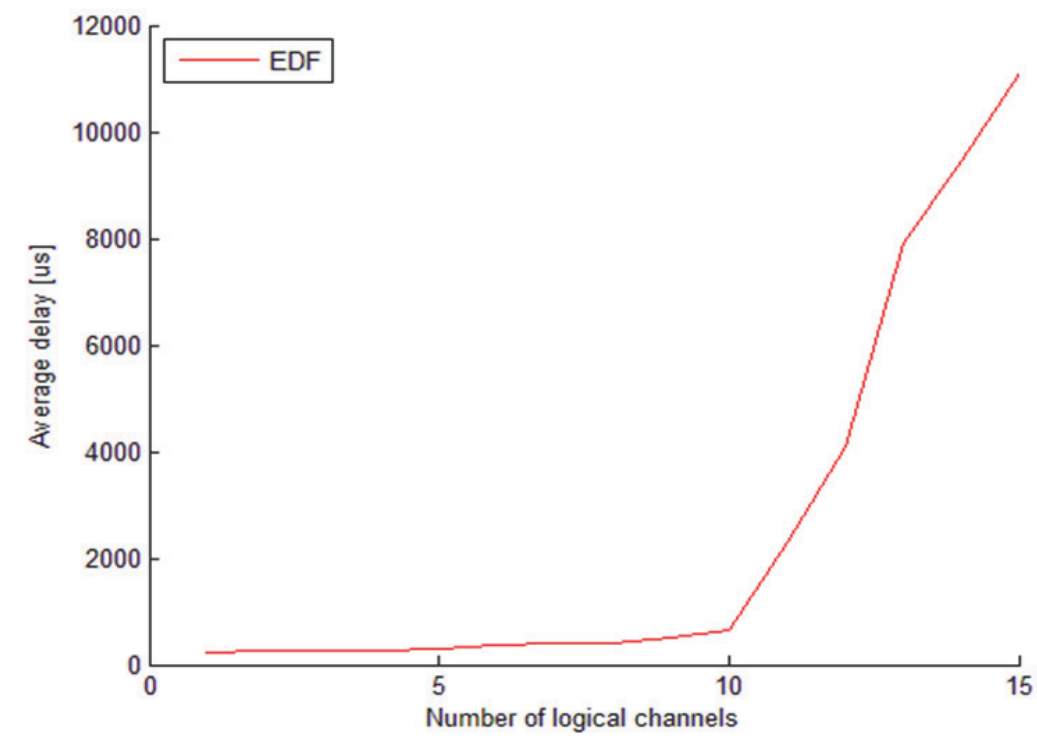

Figure 5. Average delay For DPQ (HRT) EDF 
The diagram below contains EDF and FCFS queues. With the average delay plotted against the channels/slots containing increasing traffic load. The behaviour of the delay in Figure 6 below can be explained accordingly. With an increased HRT (EDF queues) traffic intensity, the traffic class with the highest priority, HRT traffic, is affected the least, while the remaining traffic types experience a more significant increase in delay. The average delay for the NRT traffic is now plotted in the figure. As the traffic load in the system increases, the traffic experiences a slight increase in delay, while the HRT (EDF) traffic remains at a constant average delay of 2 packets per slot throughout duration of the simulation. Because of its low priority, the NRT (FCFS) traffic has to wait for the real-time traffic classes to be sent, which increases its delay considerably of the FCFS queues as more packet were enqueued. For high HRT (EDF) traffic intensity, SRT traffic gets starved and hence increases the delay and too few packets are sent to provide statistically reliable results for Figure 5

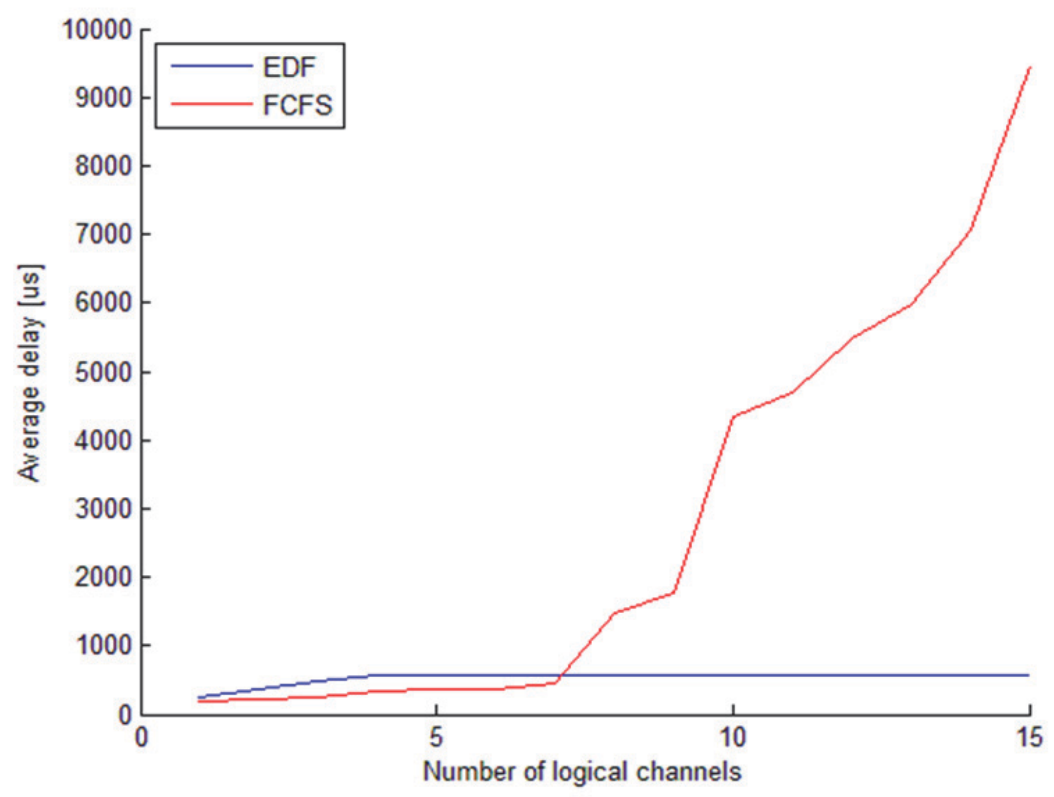

Figure 6. Average delay for FPQ(SRT) and DPQ(HRT) FCFS AND EDF respectively

With an increased HRT (hard real time traffic) intensity (Figure 6), the prioritized HRT traffic is affected the least, while the SRT traffic type experiences a higher increase in average delay. In Figure 6, the delay for the simulated FCFS traffic is plotted against a traffic probability of up to $15 \%$. The 
diagram contains values for packets with different deadlines: As expected, the delay times rise as the traffic in the networks gets denser, but a change of deadline does not influence the delay of a packet, which results in almost identical curves for the different assumed deadlines. Up to an intensity of 7 , the delay is very low for FCFS, but at intensities greater than that, the delay rises rapidly, which indicates that the network becomes saturated around a traffic density of 7 packets per node and time slot. This finding shows EDF remains in a constant average delay because of its priority. It can be concluded that EDF scheduling algorithm outperforms FCFS.

\subsection{Deadline miss ratio analysis}

First by deadline miss ratio we mean the slight different in the execution time and the known deadline and is termed Deadline miss ratio. Deadline miss ratio, $\mathrm{M}$ is the ratio of the number of missed deadline and the total number of deadline in an observable window. This can be controlled usually in EDF by changing their service level, as earlier described above.

In Figure 7 we plot the deadline miss ratio against the channels. As seen below each channels consist of 5, 10 and 15 packets per channels. Our queue consist of diverse priority queue and are been scheduled by their deadline. Below verifies that at low traffic load the deadline miss remain at Zero. But it got to a point at 8 packets per node, which the system could not actually cope and hence results in dead line miss for lower priority packets.

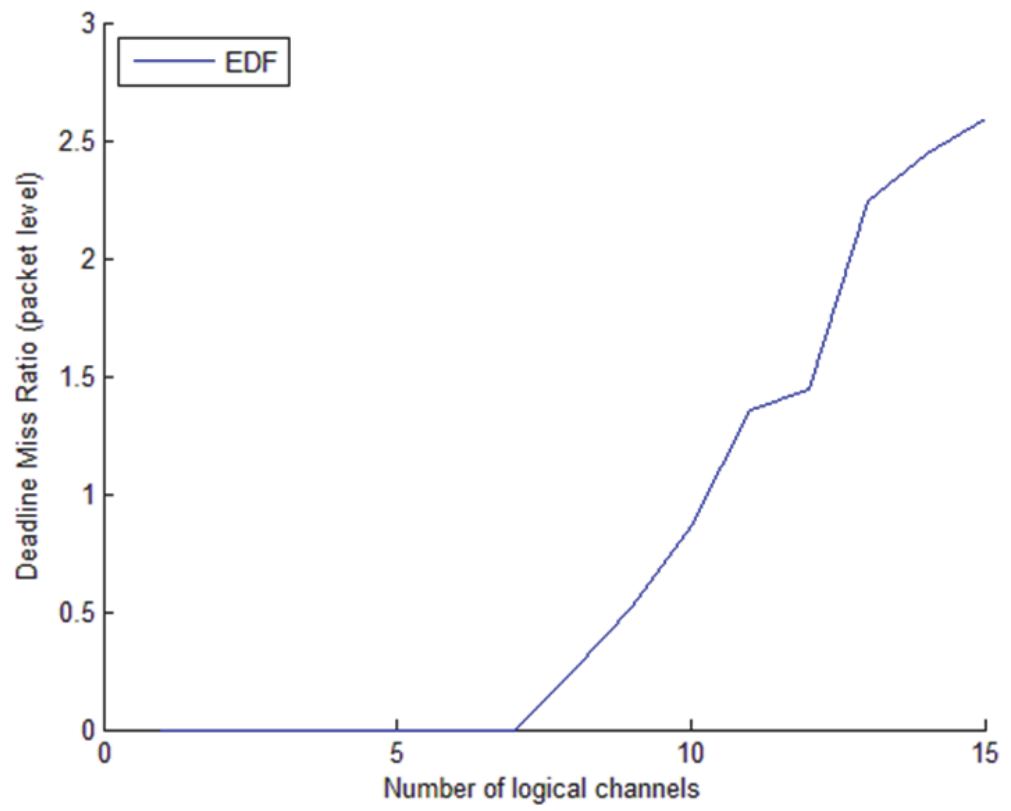

Figure 7. Deadline miss ratio for EDF 
As can be seen in Figure 8, is a graph that shows EDF and FCFS comparison in terms of deadline miss ratio. Figure 8 verifies that the HRT traffic meets all its deadline requirements. In spite of the increasing SRT traffic load, the deadline miss ratio of the HRT traffic remains zero until a traffic intensity 9 , while the SRT/FCFS deadline miss ratio increases considerably to $0.25 \%$ even at traffic load of 6 pockets per node, due to its low priority. It also verifies how slight increase in average delay at high HRT traffic intensities does not affect the HRT deadline miss ratio. This shows that the HRT capacity of the network is not challenged by the imposed traffic intensities. The sudden increase in average delay for SRT packets, as seen in Figure 8, leads to a deadline miss ratio of about 0.25 at the maximum HRT traffic intensity of 6 packets per nodes, while EDF remains at 0.1 . even at 10 packet per node, before the overload that results in a deadline miss ratio of 2.5 at a traffic load of 15 packets per node.

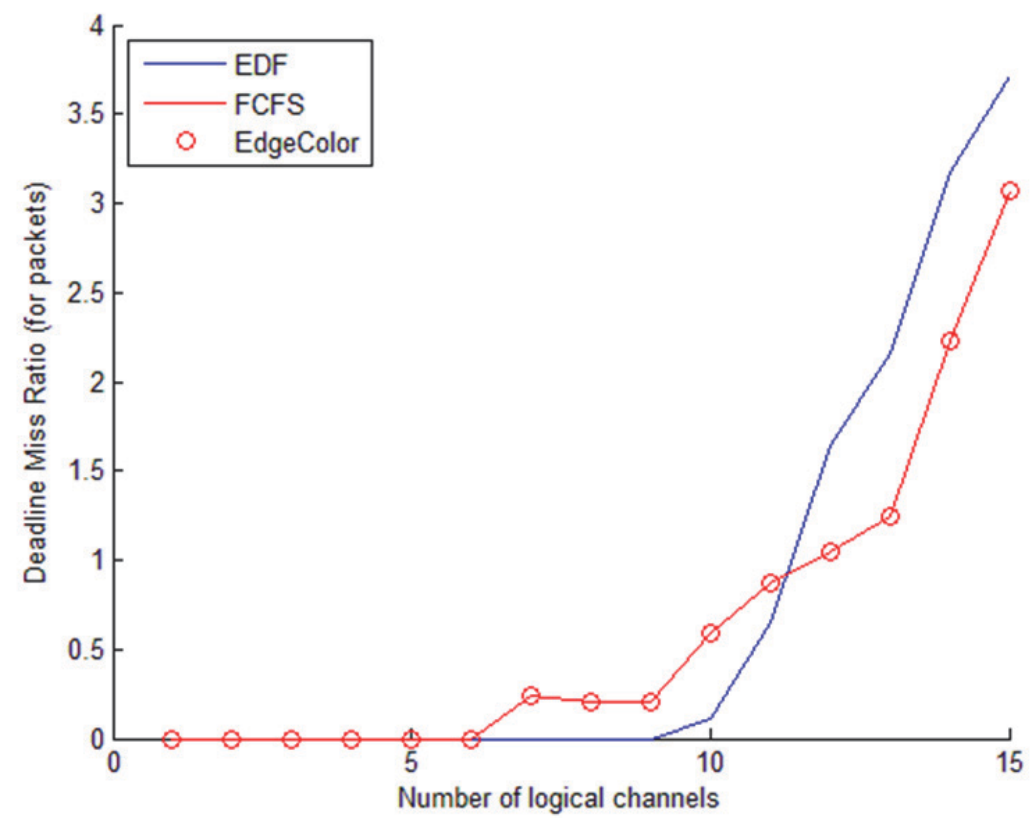

Figure 8. Deadline Miss ratio for EDF and FCFS

In conclusion, it can be said that EDF outperform FCFS in most of our simulations. The deadline miss ratio is for both policy is plotted in Figure 8, as the intensity approaches the network's saturation point, the deadline-missratio rises rapidly to almost $4 \%$ immediately. The deadline-miss-ratio is not completely independent of the deadline, as a shorter deadline results in a higher deadline-miss-ratio at lower traffic intensities. The deadline-miss-ratio 
is independent of the number of nodes connected to the network. The diagram is an additional proof of how both policy struggle for task scheduling and priority.

\section{Conclusions}

In this paper we have reviewed form and methods for real time communication in packet switched networks. We illustrate both architectural, mathematical and algorithmic aspect of real time communication traffic and non real time traffic. Model such as this will play a significant role in an era of high speed integrated network. We have revised a packet scheduling algorithm from its original proof. The importance of this algorithm lies in the fact that we can use it to deploy real-time packet switching network that supports realtime distributed applications. In our original analysis, different from the original algorithm, the EDF algorithm is considered to reason about packet orders.

\section{REFERENCES}

1. Xiaojie Li, Xianbo He, 2010, The improved EDF scheduling algorithm for embedded real-time system in the uncertain environment. Advanced Computer Theory and Engineering (ICACTE), 3rd International Conference V4-563 - V4566

2. Pachchigar P., Eswaran P.; Boke A.K., 2013, Design and implementation of deadline based EDF algorithm on ARM LPC2148, Information \& Communication Technologies (ICT), IEEE Conference. pages: 994 - 997

3. Abugchem, F.; Short, M.; Donglai Xu, 2012, A hybrid EDF algorithm for implementing resource-constrained real-time control applications, Emerging Technologies \& Factory Automation (ETFA), IEEE 17th Conference, pages: 1-4

4. Gupta A., Kohli S., Jha S., 2013, Performance of EDF-BF algorithm under QoS constraint in grid heterogeneous environment. Information Systems and Computer Networks (ISCON), International Conference, pages: 170-172. 